\title{
Review Article \\ Radical Reactions in the Gas Phase: Recent Development and Application in Biomolecules
}

\author{
Yang Gao, ${ }^{1}$ Jiexun Bu, ${ }^{2}$ Zhou Peng, ${ }^{2}$ and Biwei Yang ${ }^{2}$ \\ ${ }^{1}$ Bioanalytical Chemistry, Covance Laboratories, Inc., 3301 Kinsman Boulevard, Madison, WI 53704, USA \\ ${ }^{2}$ Department of Chemistry, Purdue University, West Lafayette, IN 47907, USA \\ Correspondence should be addressed to Yang Gao; yang.gao2@covance.com
}

Received 30 October 2014; Accepted 5 December 2014; Published 28 December 2014

Academic Editor: Lu Yang

Copyright (c) 2014 Yang Gao et al. This is an open access article distributed under the Creative Commons Attribution License, which permits unrestricted use, distribution, and reproduction in any medium, provided the original work is properly cited.

\begin{abstract}
This review summarizes recent literature describing the use of gas phase radical reactions for structural characterization of complex biomolecules other than peptides. Specifically, chemical derivatization, in-source chemical reaction, and gas phase ion/ion reactions have been demonstrated as effective ways to generate radical precursor ions that yield structural informative fragments complementary to those from conventional collision-induced dissociation (CID). Radical driven dissociation has been applied to a variety of biomolecules including peptides, nucleic acids, carbohydrates, and phospholipids. The majority of the molecules discussed in this review see limited fragmentation from conventional CID, and the gas phase radical reactions open up completely new dissociation channels for these molecules and therefore yield high fidelity confirmation of the structures of the target molecules. Due to the extensively studied peptide fragmentation, this review focuses only on nonpeptide biomolecules such as nucleic acids, carbohydrates, and phospholipids.
\end{abstract}

\section{Introduction}

The innovation and implementation of novel ion dissociation methods remain the forefront field of mass spectrometry research due to the demand to generate structural informative fragmentation patterns for better identification of a diverse array of molecules. The fundamentals of all activation methods are quite similar, in a way that they all involve depositing energy into an ion to induce bond cleavages, the fragments of which are then used to deduce the structural or sequence information of the precursor molecules. As the mass spectrometric methodologies and technologies for biological and biotechnology problems continue to rapidly evolve and improve, more versatile tools are introduced for characterization of molecules of higher complexity $[1,2]$.

The way ions fragment in the gas phase is largely determined by the ion structures, binding energies, and conformations [3-9]. The most commonly used collisional based dissociation method involves accelerating an ion to a high velocity, letting it collide with an inert gas and resulting in conversion of kinetic energy into internal energy, which then leads to fragmentation of the ion $[10,11]$. Due to the robustness and ease of implementation of the collision-induced dissociation (CID) or collision-activated dissociation (CAD), this technique became an integral part of almost every commercial tandem mass spectrometer $[12,13]$. However, as the size and complexity of the analyte continue to increase, the need for alternatives to CID has led to the development of many other dissociation techniques, including electron capture dissociation (ECD) [14], electron transfer dissociation (ETD) [15], ion-ion reactions [16, 17], and photodissociation (PD) [18-20]. Most of these techniques involve the generation of a radical site on the parent ion, and the fragmentation patterns follow an odd electron dissociation mechanism. Comparing to the common even electron fragmentation method like the CID, the radical mediated bond dissociation presents complementary structural information of the analyte of interest in many cases. For instance, ECD or ETD generates a radical site through an exothermic electron attachment process and is widely used in preserving posttranslational modifications of the peptides and proteins during large scale bottom-up or top-down proteomics applications [21].

Recently, the radical mediated dissociation technique has been expanded to fields other than peptide and proteins. 


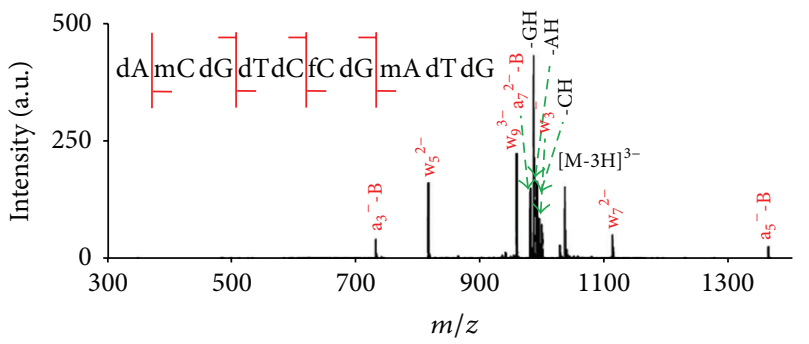

(a)

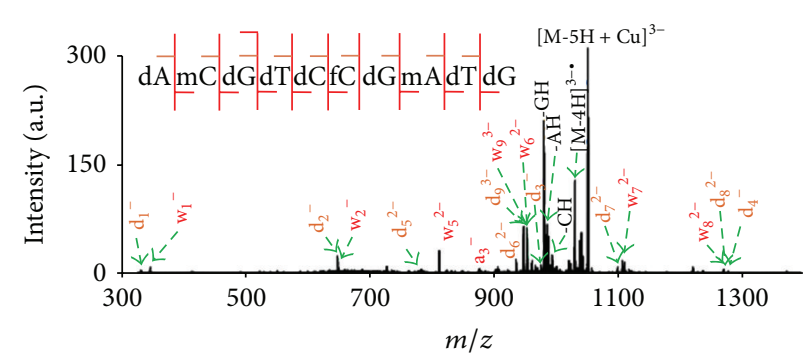

(b)

FIGURE 1: MS/MS product ion spectra of $2^{\prime}-(\mathrm{F}, \mathrm{H}, \mathrm{OMe})$ mixmer. (a) Ion trap CID of $[\mathrm{M}-3 \mathrm{H}]^{3-}$ and (b) ion trap CID of $[\mathrm{M}-4 \mathrm{H}]^{3-\bullet}$ formed from negative electron transfer reaction between $[\mathrm{M}-4 \mathrm{H}]^{4-}$ and $\mathrm{Cu}(\mathrm{phen})_{2}{ }^{+}$. Reprinted with permission from [28]. Copyright 2012 John Wiley \& Sons, Ltd.

The booming of this technique suggests the wide application of gas phase radical chemistry in biomolecular structural elucidation, including proteins, nucleic acids, glycans, and lipids. The present review is devoted to summarize the development and application of the radical mediated fragmentation and gas phase radical reaction in recent years and demonstrate their capability in biological and biotechnology researches. The focuses of the review are on nucleic acids, oligosaccharides, and lipids. Results using radical-driven dissociation on peptides were recently reviewed by $\mathrm{Oh}$ and Moon [22]. Most of the nonconventional ways of generating radical ions in the gas phase discussed in the review require specially modified instrument that is not commercially available, which provides directions for future instrumentation development that can better facilitate the biological researches.

\section{Radical Reaction of Nucleic Acids}

Structural elucidation of oligonucleotides using mass spectrometry took off ever since the introduction of electrospray [23]. CID has been the most commonly used technique in obtaining sequence informative fragments of the ion of interest. It had proved powerful and robust until recently the focus moved to chemically modified oligonucleotides [2427], which have better pharmaceutical potentials.

Gao and McLuckey [28] reported that $2^{\prime}$-substituted oligonucleotide has different fragmentation pattern compared to nonmodified DNA or RNA single strands. The gas phase stability of $2^{\prime}$-substituted nucleotide is higher than the DNA or RNA moieties, and thus the $2^{\prime}$-substituted moieties are inert to collision activation when there are DNA or RNA moieties in the same strand. Specifically, oligonucleotide "mixmers" that do not have the same 2 'modifications result in "cleavage gaps" due to significant differences in the energetic requirements associated with cleavages of the adjacent phosphodiester backbones. For instance, the presence of DNA moieties in the sequence, along with residues with $2^{\prime}$-OMe and $2^{\prime}$-fluoro substituents, can result in a situation where all of the backbone decomposition occurs via the low energy channels associated with DNA, whereas little or no structurally diagnostic cleavages take place on the $3^{\prime}$-sides of the $2^{\prime}$-O-methyl and $2^{\prime}$-fluoro substituted residues (Figure 1(a)). The gas phase stability of the $3^{\prime}$-side phosphodiester bond follows the order: $2^{\prime}$-fluoro $>2^{\prime}$ - $\mathrm{OMe}>2^{\prime}-\mathrm{OH}>2^{\prime}-\mathrm{H}$ according to a systematic study of a series of chemically modified 21- and 23-mers [29]. The McLuckey group further investigated the possibility of using radical mediated fragmentation to obtain a more universal fragmentation pattern [30]. They used copper(II) phenanthroline complex as an electron accepting reagent, which can abstract an electron from the multiply charged oligonucleotide anion during ion/ion reaction, and formed an odd electron species that bear a radical on the phosphodiester linkage (Scheme 1). Subsequent collisional activation was applied and led to further dissociation of the oligonucleotide radical anion, resulting in uniform fragments of $d / w$-ions (Figure 1(b) and Scheme 1).

In addition to radical generation via electron transfer, high energy electrons or high energy photon is also used as an approach to generate radicals on oligonucleotides. Electron detachment dissociation (EDD) takes place with higher kinetic energy electrons $(>10 \mathrm{eV})$ which cause the detachment of one or several electrons from the analytes. While ECD is typically used for multiply protonated analytes, EDD is dedicated to multiply deprotonated analytes like nucleic acids [31, 32]. Nguyen et al. [33] combined double resonance and EDD/SORI-CID experiments on thymine-rich oligonucleotides and illustrated that fragment ions originate concomitantly from both consecutive dissociation in EDD and directly from the precursor ion without electron detachment (electron induced dissociation, EID). In their work, the loss of thymine is favored by a radical process whereas it is very difficult to achieve with CID due to its lower proton affinity. Since the loss of nucleobases is the first rupture leading to the $\mathrm{a}-\mathrm{B} / \mathrm{w}$-ion series, thymine-rich DNA can yield incomplete sequence information. EDD allows the observation of w-ions from thymine-rich oligonucleotides, which are not detected in CID spectra. Ultraviolet photodissociation (UVPD) at $193 \mathrm{~nm}$ [34] and electron photodetachment dissociation (EPD) [34-36] at $260 \mathrm{~nm}$ have been applied to multiply charged oligonucleotide anions. Gabelica et al. $[35,36]$ studied the electron photodetachment of oligonucleotide anions using 250-285 $\mathrm{nm}$ laser and found that laser irradiation at $260 \mathrm{~nm}$ results in minimal fragmentation of the DNA anion, generating predominant charge-reduced radical anions. CID of the charge-reduced radical anions arising from the irradiation produced complementary a/w- and d/zfragment ions. Smith and Brodbelt [34] examined UVPD and 


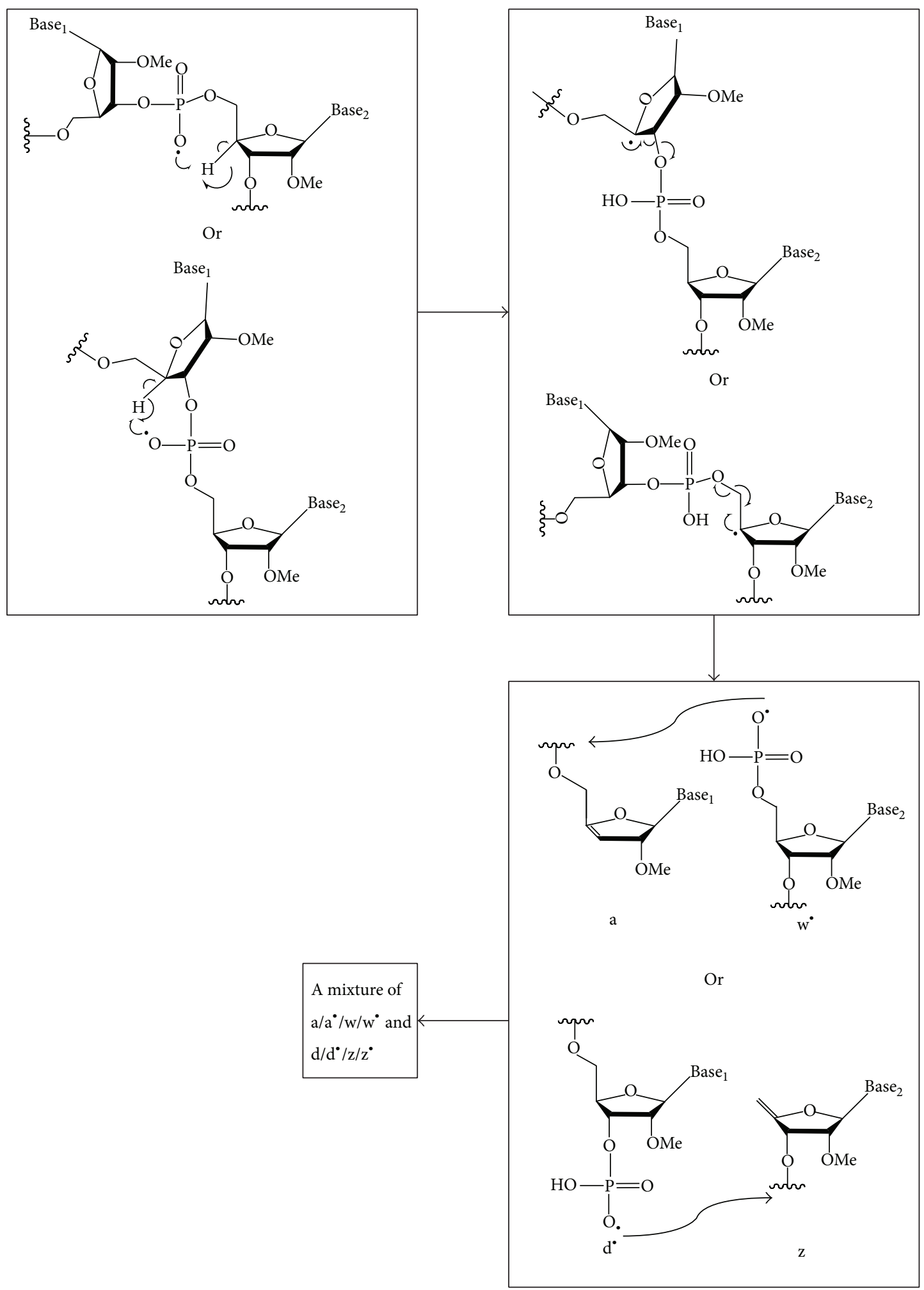

Scheme 1: Proposed pathways for phosphodiester bond cleavage following initial electron removal from the phosphodiester linkage. Reprinted with permission from [28]. Copyright 2012 John Wiley \& Sons, Ltd. 
EPD of a series of modified oligonucleotides (6- to 20-mer) at $193 \mathrm{~nm}$ and observed all types of backbone fragments and internal fragments. EPD also resulted in abundant sequence ions in terms of $\mathrm{a} /(\mathrm{a}-\mathrm{B}) / \mathrm{w}$ - and $\mathrm{d} / \mathrm{z}$-products, and it appeared even more informative than UVPD in providing similar or more complete series of fragment ions thus extending the sequence coverage up to 20-mer [35].

Generally, electrons can be removed from a multiply deprotonated oligonucleotide via electron transfer to a cation, or photodetachment. Due to the limited data published to date, it is not feasible to draw any firm conclusions regarding any differences in initial sites of radical formation or how such differences might lead to differences in structural information generated by a subsequent activation step. However, the present data do show the value of the laser photodissociation in promoting extensive arrays of $\mathrm{w}^{-}, \mathrm{z}^{-}, \mathrm{a}-$, and d-type product ions. The introduction of such hybrid techniques (e.g., activated-electron photodetachment (activated-EPD) [35]) that combine the generation of a new ion type (radical) with another structural probe has opened up new possibilities for obtaining more sequence informative fragments that can help deduce the structural information. Such hybrid methods are promising for improving the state of the art in the primary structural elucidation of nucleic acids by tandem mass spectrometry and also need further development in the near future.

\section{Radical Reaction of Oligosaccharides}

The development of mass spectrometry for oligosaccharides is relatively slow comparing to that of protein and nucleic acid analysis, largely because oligosaccharides are a more challenging set of targets for structural elucidation [37]. Conventional CID typically generates glycosidic bond cleavage [38, 39 ], whereas the more recently developed ExD techniques (ECD [40], EDD [41], and ETD [42]) provide extensive and complementary fragment information of oligosaccharide structures. A few studies have shown the application of ECD for positively charged slightly basic oligosaccharide [39, 43], whereas EDD is used primarily in the negative ion mode for analysis of acidic glycosaminoglycans (GAGs) [39], neutral, sialylated, and chloride-adducted glycans $[44,45]$. Similarly, ETD can be applied in both polarities by using different electron transfer reagents $[42,45]$.

The application of chloride anion attachment to analyze oligosaccharide was first reported by Harvey for N-linked glycans [46]. The anion attachment was implemented to simultaneously analyze neutral and acidic oligosaccharide without changing the instrument polarity. Kornacki et al. [44] employed EDD and CID for the structural elucidation of underivatized maltoheptaose bearing a chloride anion adduct. The results suggested that EDD of underivatized chloride-adducted carbohydrates yields complementary structural information to the EDD of the corresponding doubly charged species. It was noted that complete isolation of the doubly sialylated oligosaccharide was not achieved and led to the lack of unique fragment ions observed. Thus, this approach may be limited for the case in which oligosaccharides bear two or more acidic protons.
Quite a few mechanisms have been proposed for ExD, all of which involve a radical mediated fragmentation process followed by complex hydrogen migration and rearrangement $[41,43]$. The major fragmentation channels are glycosidic bond cleavages and cross-ring cleavages. The major fallback of these techniques is the fragmentation efficiency as well as the consistency of the cross-ring cleavages, largely due to the absence of well-defined sites of radical generation [47]. Gao and coworkers have recently demonstrated an approach that uses chemical derivatization of the reduced terminus for proton-catalyzed glycan sequencing (PRAGS) free radical-activated glycan sequencing (FRAGS), and this has been successfully established with a range of glycans to produce systematic and predictable cleavage processes [47] (Figure 2). This method has advantage of easy derivatization and low instrumentation requirement and can be more easily implemented to most research labs comparing to the ExD technique that would require more sophisticated instrument.

In addition to $\mathrm{ExD}$, photon-based activation techniques have also been used to generate and activate oligosaccharide radical ions. Racaud et al. [48] examined the dissociation behavior of deprotonated heparin-derived di- to tetrasaccharides under UV irradiation at $220 \mathrm{~nm}$. Two fragmentation pathways, photon-induced dissociation and electron detachment, can be observed, and the competition can be controlled by manipulating the ionization state of carboxylic groups on the oligosaccharide anions. Their work demonstrates that directed UVPD at $220 \mathrm{~nm}$ and activated-electron photodetachment (activated-EPD) can lead to more comprehensive structural analysis of oligosaccharide anions, thus complementing the conventional collisional activation modes. Oligosaccharide radical ions can also be generated by photodissociation of iodide derivatives. Zhang and Julian [49] covalently or noncovalently attached a radical-generating reagent to an oligosaccharide ion, and a radical can be generated by homolytic bond cleavage of specific carboniodine bonds in protonated systems by UVPD followed by radical transfer from the reagent to the analyte. Subsequent activation of the radical species generates information-rich spectra including a variety of cross-ring fragments. Their work demonstrates the advantage of radical chemistry over other approaches for the characterization of oligosaccharides, especially the identification of positional isomers of various sizes.

\section{Radical Reaction of Lipids}

Structural characterization of lipids is almost always limited to conventional tandem mass spectrometry, which is sufficient for determining lipid class and identifying the number of carbons as well as the degree of unsaturation. However, the more detailed structural information, including double bond position, chain branching, and cyclic structures, is beyond the capability of conventional ion trap CID and beam type CID. The dissociation of even electron lipid ions does not generate product ions from internal fragmentation of the fatty acyl chains. In most cases, the fatty acyl chains "fall off" as neutrals and are therefore not detected by the mass spectrometer. Previous studies have shown that intrachain 


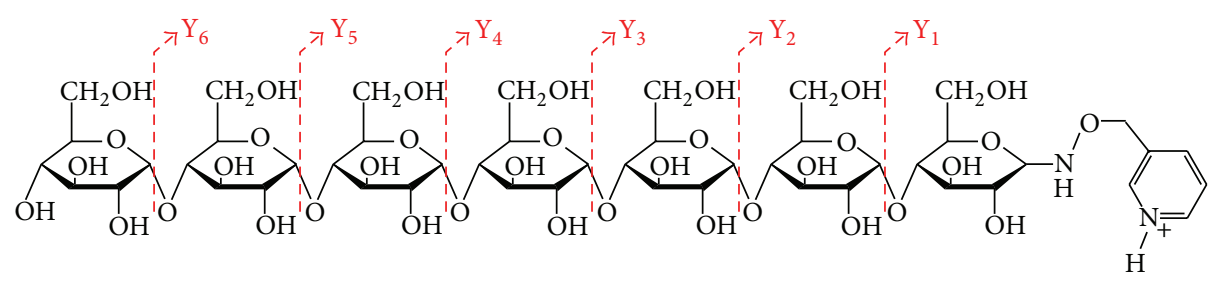

(a)

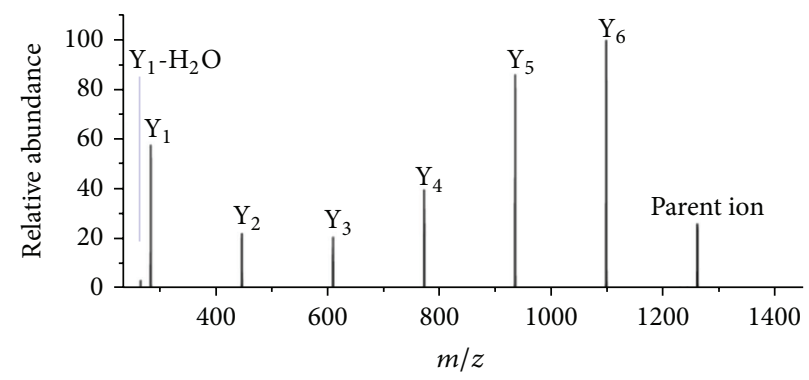

(b)

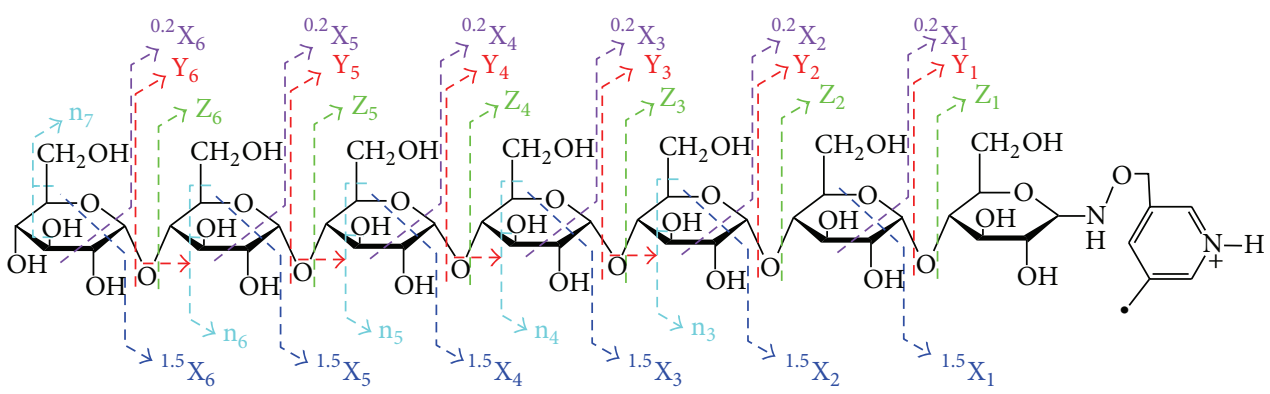

(c)

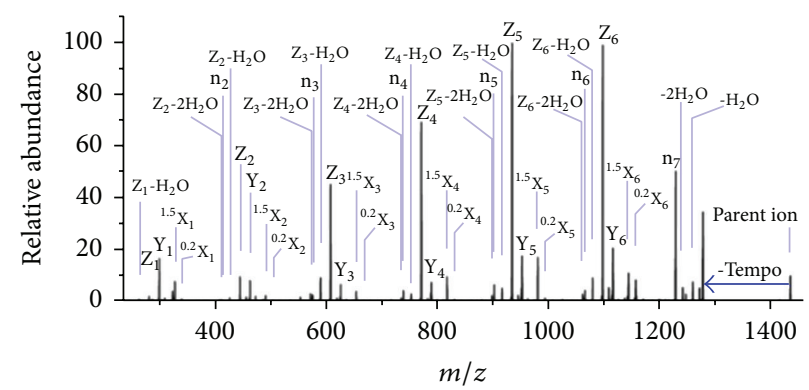

(d)

FIGURE 2: Fragmentation patterns observed following CID of singly protonated PRAGS-derivatized (a) and FRAGS-derivatized (c) maltoheptaose and CID spectra of singly protonated PRAGS-derivatized (b) and FRAGS-derivatized (d) maltoheptaose. Reprinted with permission from [47]. Copyright 2013 American Chemical Society.

fragmentation of lipids can be achieved by either high energy CID (>keV) or multistage CID on ion-trap platforms. The former requires using sectors or TOF/TOF instrument [50, 51], whereas the latter takes place on ion-trap platforms $[52,53]$. The broader implementation of these techniques has been limited primarily due to the incompatibility of the high energy platforms with ESI and the low sensitivity of the $\mathrm{MS}^{n}$ experiments [54]. Castro-Perez and coworkers [55] demonstrated the use of time-aligned-CID spectra on an ionmobility device coupled to a quadrupole time-of-flight mass spectrometer (IM-qTOF MS) to improve the sensitivity of the multistage CID.
Alternatively, selective ion-molecule reactions in the gas phase (e.g., covalent attachment chemical ionization [56] and ozone induced dissociation [57]) have been utilized to pinpoint the double bond positions in complex lipids. Dobson and Christie [58] found that CID of radical ions produced by traditional electron impact ionization (EI) resulted in extensive intrachain fragmentation in fatty acids and their derivatives. However, the use of EI is limited to complex lipids due to their high vapor pressure and thermal instability. In order to take advantage of the radical mediated fragmentation without being limited to EI, electron transfer reactions have been applied to lipids $[59,60]$ analogous to 
the contemporary methods used in the proteomics community. These approaches are also limited in lipidomics because most lipids produce predominantly singly charged ions.

Pham and coworkers [54] took an alternative way to generate radical ions by attaching a photocaged radical precursor to the analytical target and then "uncage" the radical by photon irradiation in the mass spectrometer. The subsequent CID of the nascent radical ion induced radical mediated dissociation of the precursor. Two photocaged radical precursors (4-iodobenzoate (IB) and 4-iodoaniline (IA)) have been investigated as they bear a UV-labile carboniodine bond and a para-substituted functional group that can bind noncovalently to complex lipids. The radical mediated dissociation spectra (Figure 3) show a variety of fragments arising from acyl chain backbone cleavages. The application of radical mediated dissociation is also demonstrated for the biologically derived lipid extracts.

Instead of UV irradiation inside a mass spectrometer, $\mathrm{Ma}$ and coworkers [61] induced the radical reaction in the ESI source (Figure 4(a)). They demonstrated an approach that couples tandem mass spectrometry with the unique chemistry of Paternò-Büchi $(\mathrm{P}-\mathrm{B})$ reaction towards $\mathrm{C}=\mathrm{C}$ bond for lipid structure elucidation. The online $\mathrm{P}-\mathrm{B}$ reaction was conducted using acetone as the reagent and reacting with oleic acid. The UV irradiation at the source resulted in a mass increase of $58.0423 \mathrm{Da}$ relative to deprotonated oleic acid, corresponding to $\mathrm{C}_{3} \mathrm{H}_{6} \mathrm{O}$, the elemental composition of acetone (Figures 4(b) and 4(e)). CID of the $\mathrm{C}_{3} \mathrm{H}_{6} \mathrm{O}$ adducted precursor resulted in all retro $\mathrm{P}-\mathrm{B}$ pathways, which revealed the location of the double bonds in oleic acid. This approach was also investigated in the context of complex phospholipids and successfully yielded diagnostic ions for double bond positions. This technique has simple experimental setup and does not need MS instrument modifications, which makes it accessible and attractive to many laboratories.

\section{Conclusion}

The development of mass spectrometry instrumentation is always challenged by the fast growing demand from the biological researches. The complexity of the target molecule and the level of structure information require not only more sensitive and accurate MS instrument, but also more specific probes that can "tease out" the structural information in a predictable manner. Collision-induced dissociation, although being the most powerful and robust tool, starts to see its limitations in many different fields due to its inherent energy deposition mechanism and the gas phase fragmentation chemistry of biological ions. As the only information that comes out of a mass spectrometer is the

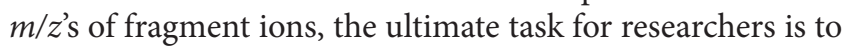
obtain as many types of predictable fragment ions as possible. This inevitably makes any single probe (collision with the inert neutral particles) not feasible. The invention of the electron based techniques significantly improves the amount of information that a mass spectrum can provide. However, they suffered from low efficiency and sometimes unpredictable fragmentation pattern. Extensive researches have been done on various ways of adding or substracting an electron from

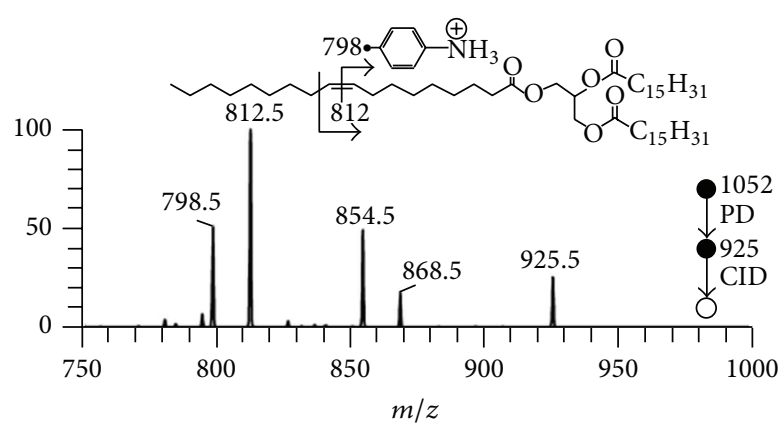

(c)
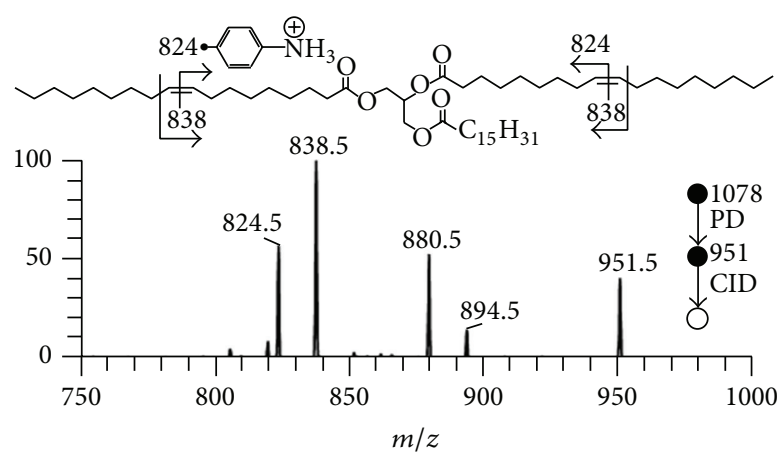

(d)
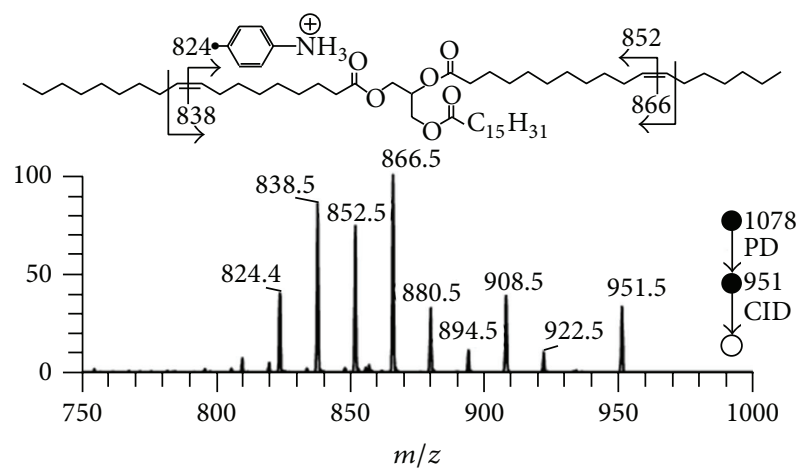

(e)

FIGURE 3: Radical mediated dissociation spectra obtained from $[\mathrm{TG}+\mathrm{IA}]^{+}$complexes for (c) TG(16:0/16:0/9Z-18:1), (d) TG(16:0/ 9Z-18:1/9Z-18:1), and (e) TG(16:0/11Z-18:1/9Z-18:1). Adapted with permission from [54], (a) and (b) not included. Copyright 2012 American Chemical Society.

the target ion using a wide range of deposit energies. The fragmentation pattern largely depends on how much energy is put into the system, how much energy is converted into internal energy, and how fast the conversion takes place. Then photodissociation was developed to make faster and more accurate deposition of the energy. Of all the new techniques, the generation of radical is almost always being the key in the fragmentation process. Observations made by many research groups over the past decades have been broadly selfconsistent. However, some inconsistencies in the literature have been observed, which may arise from the differences in instrument platforms and variations in conditions. For example, different correlations between electron photodetachment efficiency and nucleobase identification have been reported. 


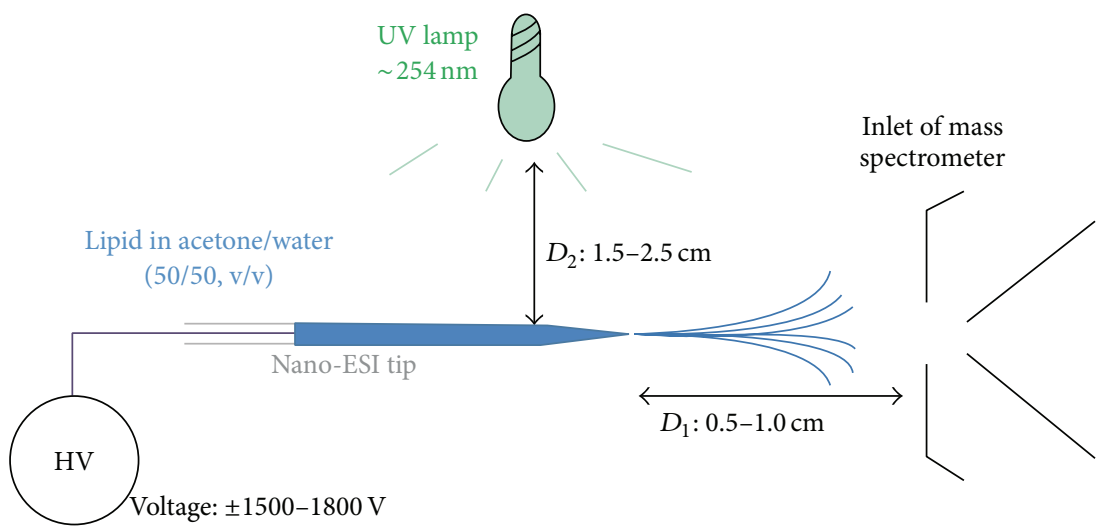

(a)

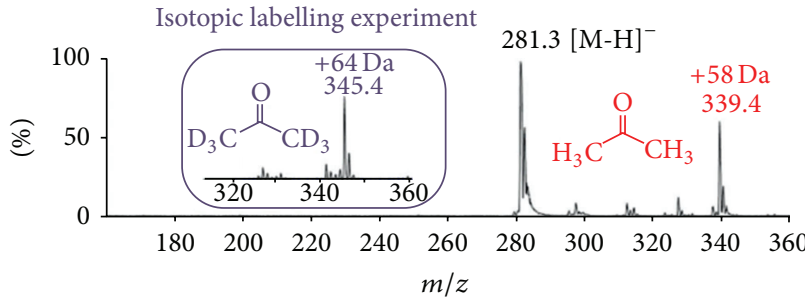

(b)

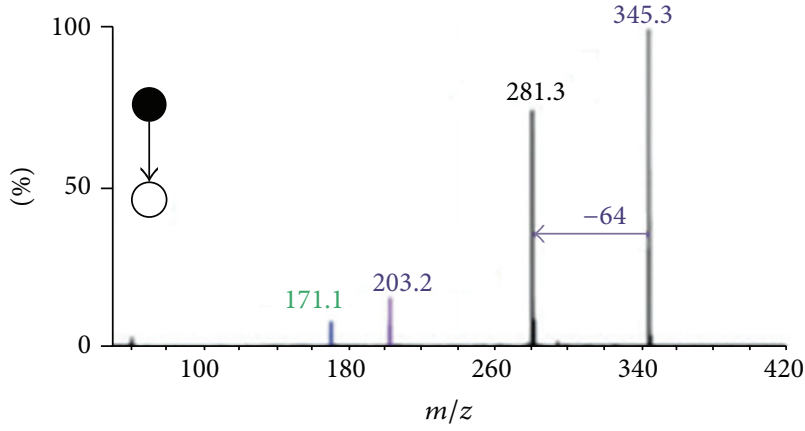

(d)

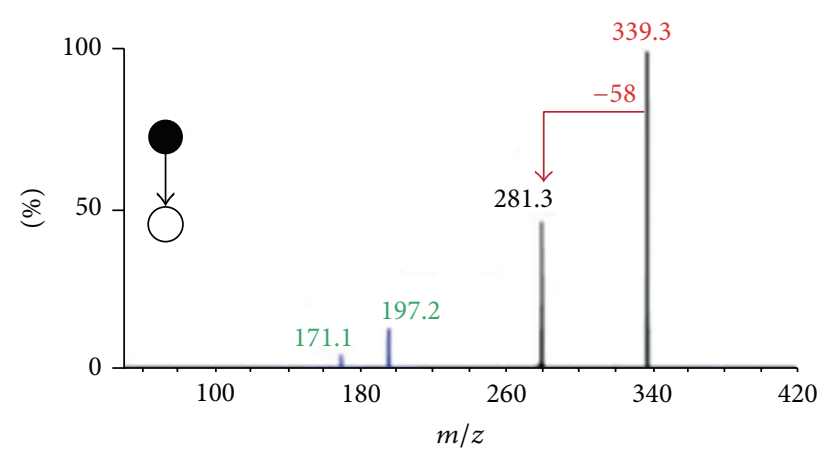

(c)
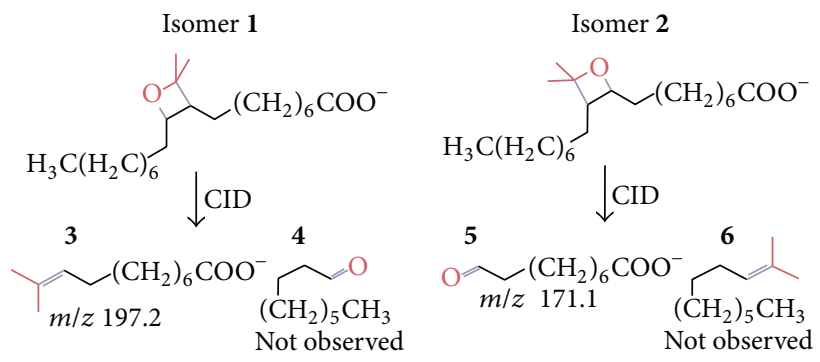

(e)

FIgURE 4: Online coupling of P-B reactions with MS for lipid analysis. (a) Experimental setup. (b) P-B reaction mass spectrum of oleic acid and acetone induced by UV irradiation of nano-ESI. Inset: P-B reaction spectrum using D6-acetone (C3D6O). MS2 CID of the P-B reaction products at (c) $m / z 339.3$ and (d) $m / z$ 345.3. (e) Fragmentation scheme of P-B reaction product isomers. Reprinted with permission from [61]. Copyright 2014 Wiley-VCH Verlag GmbH \& Co. KGaA, Weinheim.

As a tool for the structural characterization of biomolecules, tandem mass spectrometry continues to evolve and versatile applications are being developed to fit the needs of various analyses.

This review mainly focuses on different ways of generating radical ion precursors in the gas phase and the application of radical reactions in structural elucidation of biomolecules. The contents cover solution phase derivatization, in-source derivatization, and gas phase ion/ion reactions. These approaches involve different levels of protocol complexity and require various types of instrument modification. Regardless of immediate application to the field, the methods presented all yield extensive structural information and have unique potential applications for specific analytes. Nevertheless, the sample preparation protocols/new instrumentation need to be further optimized to fit the general demand for the larger community. For instance, the solution derivatization of glycans needs simpler preparation procedures that can ideally be designed as a kit. Moreover, the in-source $\mathrm{P}-\mathrm{B}$ reaction needs better parameter control to be able to fit the quantitation needs of an LC-MS platform. Similarly, ideal electron transfer reagent needs further investigation for modified oligonucleotides. With increasing discoveries made on the fundamental gas phase radical 
reactions, more applications will be developed and better fidelity and specificity will be achieved.

\section{Conflict of Interests}

The authors declare that there is no conflict of interests regarding the publication of this paper.

\section{References}

[1] J. R. Yates, C. I. Ruse, and A. Nakorchevsky, "Proteomics by mass spectrometry: approaches, advances, and applications," Annual Review of Biomedical Engineering, vol. 11, pp. 49-79, 2009.

[2] R. J. Mishur and S. L. Rea, "Applications of mass spectrometry to metabolomics and metabonomics: detection of biomarkers of aging and of age-related diseases," Mass Spectrometry Reviews, vol. 31, no. 1, pp. 70-95, 2012.

[3] J. S. Brodbelt, "Photodissociation mass spectrometry: new tools for characterization of biological molecules," Chemical Society Reviews, vol. 43, no. 8, pp. 2757-2783, 2014.

[4] I. K. Webb, Y. Gao, F. A. Londry, and S. A. McLuckey, “Trapping mode dipolar DC collisional activation in the RF-only ion guide of a linear ion trap/time-of-flight instrument for gaseous bioion declustering," Journal of Mass Spectrometry, vol. 48, no. 9, pp. 1059-1065, 2013.

[5] Z. Cao, W. P. Forrest, Y. Gao, P. E. Fanwick, Y. Zhang, and T. Ren, "New Iron(III) bis(acetylide) compounds based on the iron cyclam motif," Inorganic Chemistry, vol. 50, no. 16, pp. 73647366, 2011.

[6] Z. Cao, W. P. Forrest, Y. Gao, P. E. Fanwick, and T. Ren, "Trans- $\left[\mathrm{Fe}(\text { cyclam })\left(\mathrm{C}_{2} \mathrm{R}\right)_{2}\right]^{+}$: a new family of iron(III) bisalkynyl compounds," Organometallics, vol. 31, no. 17, pp. 61996206, 2012.

[7] Z. Cao, P. E. Fanwick, W. P. Forrest, Y. Gao, and T. Ren, “New $\mathrm{Fe}(\mathrm{III})(\mathrm{cyclam})$ complexes bearing axially bound geminaldiethynylethenes," Organometallics, vol. 32, no. 16, pp. 46844689, 2013.

[8] J. Savchenko, P. E. Fanwick, H. Hope, Y. Gao, C. K. Yerneni, and T. Ren, "New diruthenium (II,III) compounds bearing terminal olefin groups," Inorganica Chimica Acta, vol. 396, pp. 144-148, 2013.

[9] Z. Cao, B. Xi, D. S. Jodoin et al., "Diruthenium-polyyn-diyldiruthenium wires: electronic coupling in the long distance regime," Journal of the American Chemical Society, vol. 136, no. 34, pp. 12174-12183, 2014.

[10] A. Li, H. Wang, Z. Ouyang, and R. G. Cooks, "Paper spray ionization of polar analytes using non-polar solvents," Chemical Communications, vol. 47, no. 10, pp. 2811-2813, 2011.

[11] A. Li, P. Wei, H.-C. Hsu, and R. G. Cooks, "Direct analysis of 4-methylimidazole in foods using paper spray mass spectrometry," Analyst, vol. 138, no. 16, pp. 4624-4630, 2013.

[12] L. Sleno and D. A. Volmer, "Ion activation methods for tandem mass spectrometry," Journal of Mass Spectrometry, vol. 39, no. 10, pp. 1091-1112, 2004.

[13] A. Li, Q. Luo, S.-J. Park, and R. G. Cooks, "Synthesis and catalytic reactions of nanoparticles formed by electrospray ionization of coinage metals," Angewandte Chemie, vol. 53, no. 12, pp. 3147-3150, 2014.
[14] R. A. Zubarev, A. R. Zubarev, and M. M. Savitski, "Electron capture/transfer versus collisionally activated/induced dissociations: solo or duet?" Journal of the American Society for Mass Spectrometry, vol. 19, no. 6, pp. 753-761, 2008.

[15] J. E. P. Syka, J. J. Coon, M. J. Schroeder, J. Shabanowitz, and D. F. Hunt, "Peptide and protein sequence analysis by electron transfer dissociation mass spectrometry," Proceedings of the National Academy of Sciences of the United States of America, vol. 101, no. 26, pp. 9528-9533, 2004.

[16] S. A. McLuckey and T.-Y. Huang, "Ion/ion reactions: new chemistry for analytical MS," Analytical Chemistry, vol. 81, no. 21, pp. 8669-8676, 2009.

[17] Z. Peng, W. M. McGee, J. Bu, N. Z. Barefoot, and S. A. McLuckey, "Gas phase reactivity of carboxylates with $N$ hydroxysuccinimide esters," Journal of the American Society for Mass Spectrometry, 2014.

[18] J. P. Reilly, "Ultraviolet photofragmentation of biomolecular ions," Mass Spectrometry Reviews, vol. 28, no. 3, pp. 425-447, 2009.

[19] T. Ly and R. R. Julian, "Ultraviolet photodissociation: developments towards applications for mass-spectrometry-based proteomics," Angewandte Chemie, vol. 48, no. 39, pp. 7130-7137, 2009.

[20] R. Antoine, J. Lemoine, and P. Dugourd, "Electron photodetachment dissociation for structural characterization of synthetic and bio-polymer anions," Mass Spectrometry Reviews, vol. 33, no. 6, pp. 501-522, 2014.

[21] R. A. Zubarev, "Electron-capture dissociation tandem mass spectrometry," Current Opinion in Biotechnology, vol. 15, no. 1, pp. 12-16, 2004.

[22] H. B. Oh and B. Moon, "Radical-driven peptide backbone dissociation tandem mass spectrometry," Mass Spectrometry Reviews, 2014.

[23] J. Wu and S. A. McLuckey, "Gas-phase fragmentation of oligonucleotide ions," International Journal of Mass Spectrometry, vol. 237, no. 2-3, pp. 197-241, 2004.

[24] L. L. Cummins, S. R. Owens, L. M. Risen et al., "Characterization of fully $2^{\prime}$-modified oligoribonucleotide hetero- and homoduplex hybridization and nuclease sensitivity," Nucleic Acids Research, vol. 23, no. 11, pp. 2019-2024, 1995.

[25] C. Wetzel, S. Li, and P. A. Limbach, "Metabolic de-isotoping for improved LC-MS characterization of modified RNAs," Journal of the American Society for Mass Spectrometry, vol. 25, no. 7, pp. 1114-1123, 2014.

[26] S. Li and P. A. Limbach, "Mass spectrometry sequencing of transfer ribonucleic acids by the comparative analysis of RNA digests (CARD) approach," Analyst, vol. 138, no. 5, pp. 13861394, 2013.

[27] S. Li and P. A. Limbach, "Method for comparative analysis of ribonucleic acids using isotope labeling and mass spectrometry," Analytical Chemistry, vol. 84, no. 20, pp. 8607-8613, 2012.

[28] Y. Gao and S. A. McLuckey, "Collision-induced dissociation of oligonucleotide anions fully modified at the $2^{\prime}$-position of the ribose: $2^{\prime}-\mathrm{F} / \mathrm{-H}$ and $2^{\prime}-\mathrm{F} /-\mathrm{H} /-\mathrm{OMe}$ mix-mers," Journal of Mass Spectrometry, vol. 47, no. 3, pp. 364-369, 2012.

[29] Y. Gao, J. Yang, M. T. Cancilla, F. Meng, and S. A. McLuckey, "Top-down interrogation of chemically modified oligonucleotides by negative electron transfer and collision induced dissociation," Analytical Chemistry, vol. 85, no. 9, pp. 4713-4720, 2013. 
[30] Y. Gao and S. A. McLuckey, "Electron transfer followed by collisioninduced dissociation (NET-CID) for generating sequence information from backbonemodified oligonucleotide anions," Rapid Communications in Mass Spectrometry, vol. 27, no. 1, pp. 249-257, 2013.

[31] J. Yang, J. Mo, J. T. Adamson, and K. Håkansson, "Characterization of oligodeoxynucleotides by electron detachment dissociation fourier transform ion cyclotron resonance mass spectrometry," Analytical Chemistry, vol. 77, no. 6, pp. 18761882, 2005.

[32] J. Yang and K. Hakansson, "Characterization of oligonucleotide fragmentation pathways in infrared multiphoton dissociaton and electron detachment dissociation by Fourier transform ion cyclotron double resonance," Journal of Mass Spectrometry, vol. 15, pp. 293-304, 2009.

[33] V. H. Nguyen, C. Afonso, and J.-C. Tabet, "Concomitant EDD and EID of DNA evidenced by MSn and double resonance experiments," International Journal of Mass Spectrometry, vol. 301, no. 1-3, pp. 224-233, 2011.

[34] S. I. Smith and J. S. Brodbelt, "Characterization of oligodeoxynucleotides and modifications by $193 \mathrm{~nm}$ Photodissociation and electron photodetachment dissociation," Analytical Chemistry, vol. 82, no. 17, pp. 7218-7226, 2010.

[35] V. Gabelica, T. Tabarin, R. Antoine et al., "Electron photodetachment dissociation of DNA polyanions in a quadrupole ion trap mass spectrometer," Analytical Chemistry, vol. 78, no. 18, pp. 6564-6572, 2006.

[36] V. Gabelica, F. Rosu, T. Tabarin et al., "Base-dependent electron photodetachment from negatively charged DNA strands upon 260-nm laser irradiation," Journal of the American Chemical Society, vol. 129, no. 15, pp. 4706-4713, 2007.

[37] M. J. Kailemia, L. R. Ruhaak, C. B. Lebrilla, and I. J. Amster, "Oligosaccharide analysis by mass spectrometry: a review of recent developments," Analytical Chemistry, vol. 86, no. 1, pp. 196-212, 2014.

[38] D. J. Harvey, "Ionization and collision-induced fragmentation of N-linked and related carbohydrates using divalent cations," Journal of the American Society for Mass Spectrometry, vol. 12, no. 8, pp. 926-937, 2001.

[39] J. T. Adamson and K. Hakansson, "Electron capture dissociation of oligosaccharides ionized with alkali, alkaline earth, and transition metals," Analytical Chemistry, vol. 79, no. 7, pp. 29012910, 2007.

[40] B. A. Budnik, K. F. Haselmann, Y. N. Elkin, V. I. Gorbach, and R. A. Zubarev, "Applications of electron-ion dissociation reactions for analysis of polycationic chitooligosaccharides in Fourier transform mass spectrometry," Analytical Chemistry, vol. 75, no. 21, pp. 5994-6001, 2003.

[41] J. J. Wolff, I. J. Amster, L. Chi, and R. J. Linhardt, "Electron detachment dissociation of glycosaminoglycan tetrasaccharides," Journal of the American Society for Mass Spectrometry, vol. 18, no. 2, pp. 234-244, 2007.

[42] L. Han and C. E. Costello, "Electron transfer dissociation of milk oligosaccharides," Journal of the American Society for Mass Spectrometry, vol. 22, no. 6, pp. 997-1013, 2011.

[43] C. Zhao, B. Xie, S.-Y. Chan, C. E. Costello, and P. B. O'Connor, "Collisionally activated dissociation and electron capture dissociation provide complementary structural information for branched permethylated oligosaccharides," Journal of the American Society for Mass Spectrometry, vol. 19, no. 1, pp. 138-150, 2008.
[44] J. R. Kornacki, J. T. Adamson, and K. Håkansson, "Electron detachment dissociation of underivatized chloride-adducted oligosaccharides," Journal of the American Society for Mass Spectrometry, vol. 23, no. 11, pp. 2031-2042, 2012.

[45] J. J. Wolff, F. E. Leach, T. N. Laremore et al., "Negative electron transfer dissociation of glycosaminoglycans," Analytical Chemistry, vol. 82, no. 9, pp. 3460-3466, 2010.

[46] D. J. Harvey, "Fragmentation of negative ions from carbohydrates: part 1. Use of nitrate and other anionic adducts for the production of negative ion electrospray spectra from Nlinked carbohydrates," Journal of the American Society for Mass Spectrometry, vol. 16, no. 5, pp. 622-630, 2005.

[47] J. Gao, D. A. Thomas, C. H. Sohn, and J. L. Beauchamp, "Biomimetic reagents for the selective free radical and acidbase chemistry of glycans: application to glycan structure determination by mass spectrometry," Journal of the American Chemical Society, vol. 135, no. 29, pp. 10684-10692, 2013.

[48] A. Racaud, R. Antoine, P. Dugourd, and J. Lemoine, "Photoinduced dissociation of heparin-derived oligosaccharides controlled by charge location," Journal of the American Society for Mass Spectrometry, vol. 21, no. 12, pp. 2077-2084, 2010.

[49] X. Zhang and R. R. Julian, "Radical mediated dissection of oligosaccharides," International Journal of Mass Spectrometry, vol. 372, pp. 22-28, 2014.

[50] C. Cheng, M. L. Gross, and E. Pittenauer, "Complete structural elucidation of triacylglycerols by tandem sector mass spectrometry," Analytical Chemistry, vol. 70, no. 20, pp. 4417-4426, 1998.

[51] E. Pittenauer and G. Allmaier, "The Renaissance of High-energy CID for structural elucidation of complex lipids: MALDITOF/RTOF-MS of alkali cationized triacylglycerols," Journal of the American Society for Mass Spectrometry, vol. 20, no. 6, pp. 1037-1047, 2009.

[52] F.-F. Hsu and J. Turk, "Structural characterization of unsaturated glycerophospholipids by multiple-stage linear ion-trap mass spectrometry with electrospray ionization," Journal of the American Society for Mass Spectrometry, vol. 19, no. 11, pp. 16811691, 2008.

[53] F.-F. Hsu and J. Turk, "Electrospray ionization multiple-stage linear ion-trap mass spectrometry for structural elucidation of triacylglycerols: assignment of fatty acyl groups on the glycerol backbone and location of double bonds," Journal of the American Society for Mass Spectrometry, vol. 21, no. 4, pp. 657$669,2010$.

[54] H. T. Pham, T. Ly, A. J. Trevitt, T. W. Mitchell, and S. J. Blanksby, "Differentiation of complex lipid isomers by radical-directed dissociation mass spectrometry," Analytical Chemistry, vol. 84, no. 17, pp. 7525-7532, 2012.

[55] J. Castro-Perez, T. P. Roddy, N. M. M. Nibbering et al., "Localization of fatty acyl and double bond positions in phosphatidylcholines using a dual stage CID fragmentation coupled with ion mobility mass spectrometry," Journal of the American Society for Mass Spectrometry, vol. 22, no. 9, pp. 1552-1567, 2011.

[56] Y. Xu and J. T. Brenna, "Atmospheric pressure covalent adduct chemical ionization tandem mass spectrometry for double bond localization in monoene- and diene-containing triacylglycerols," Analytical Chemistry, vol. 79, no. 6, pp. 2525-2536, 2007.

[57] M. C. Thomas, T. W. Mitchell, D. G. Harman, J. M. Deeley, J. R. Nealon, and S. J. Blanksby, "Ozone-induced dissociation: elucidation of double bond position within mass-selected lipid ions," Analytical Chemistry, vol. 80, no. 1, pp. 303-311, 2008. 
[58] G. Dobson and W. W. Christie, "Mass spectrometry of fatty acid derivatives," European Journal of Lipid Science and Technology, vol. 104, no. 1, pp. 36-43, 2002.

[59] P. F. James, M. A. Perugini, and R. A. J. O’Hair, “Electron capture dissociation of complexes of diacylglycerophosphocholine and divalent metal ions: Competition between charge reduction and radical induced phospholipid fragmentation," Journal of the American Society for Mass Spectrometry, vol. 19, no. 7, pp. 978986, 2008.

[60] H. J. Yoo and K. Håkansson, "Determination of double bond location in fatty acids by manganese adduction and electron induced dissociation," Analytical Chemistry, vol. 82, no. 16, pp. 6940-6946, 2010.

[61] X. Ma and Y. Xia, "Pinpointing double bonds in lipids by paternò-büchi reactions and mass spectrometry," Angewandte Chemie - International Edition, vol. 53, no. 10, pp. 2592-2596, 2014. 

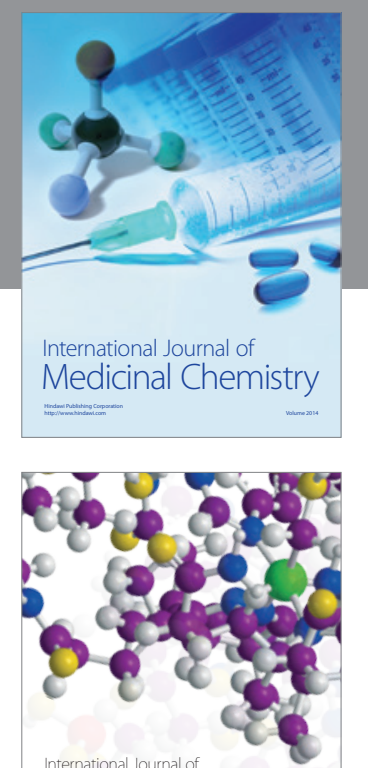

\section{Carbohydrate} Chemistry

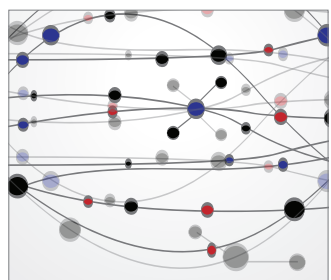

The Scientific World Journal
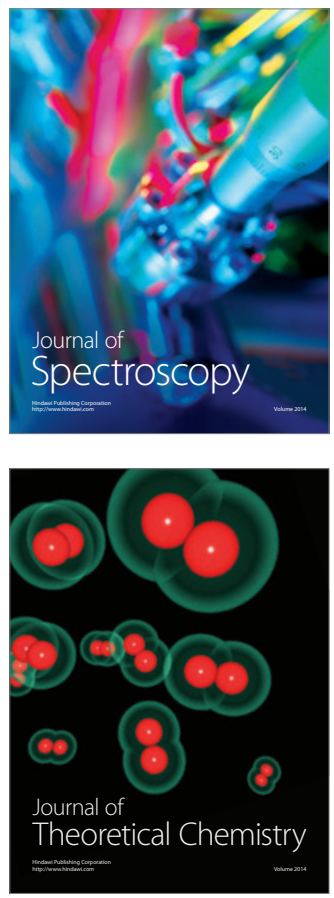
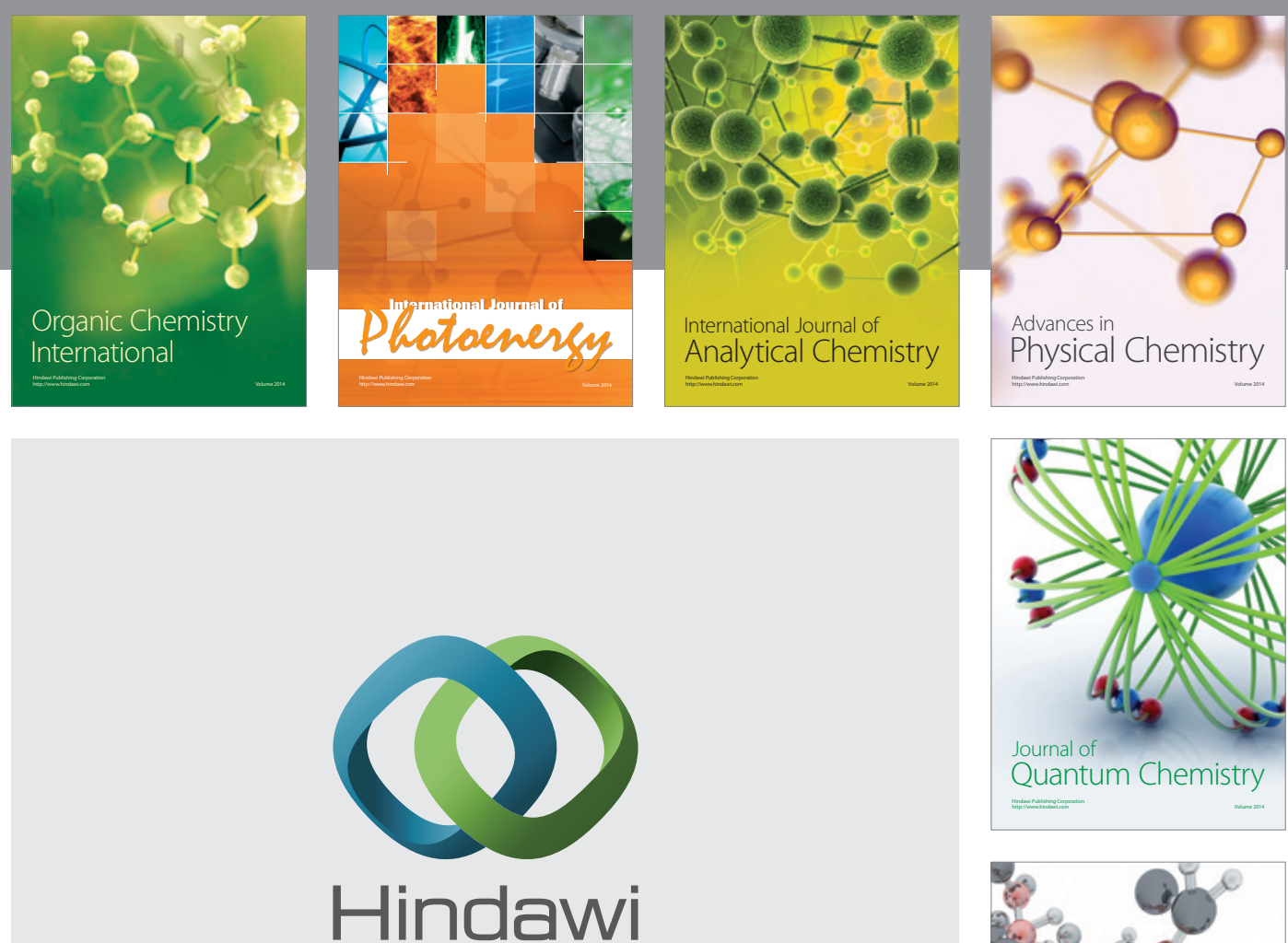

Submit your manuscripts at

http://www.hindawi.com

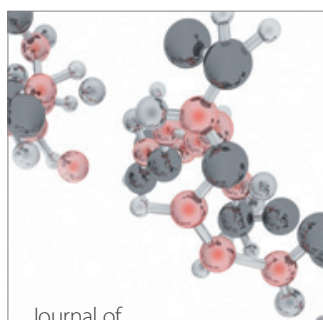

Analytical Methods

in Chemistry

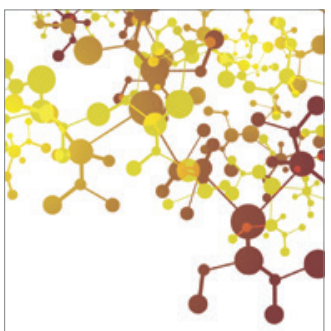

Journal of

Applied Chemistry

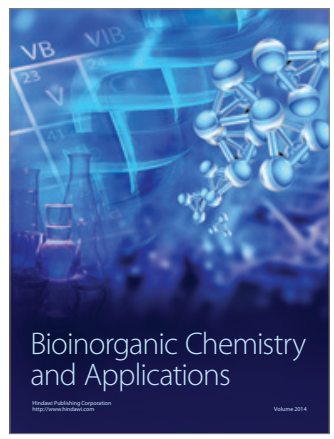

Inorganic Chemistry
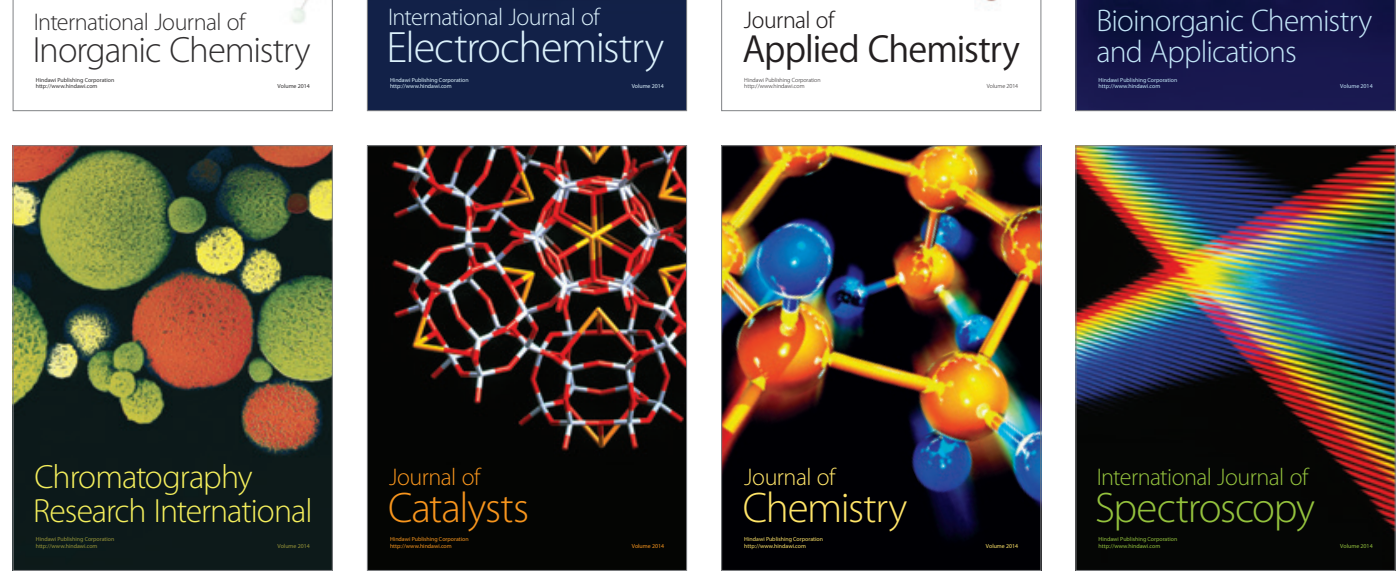